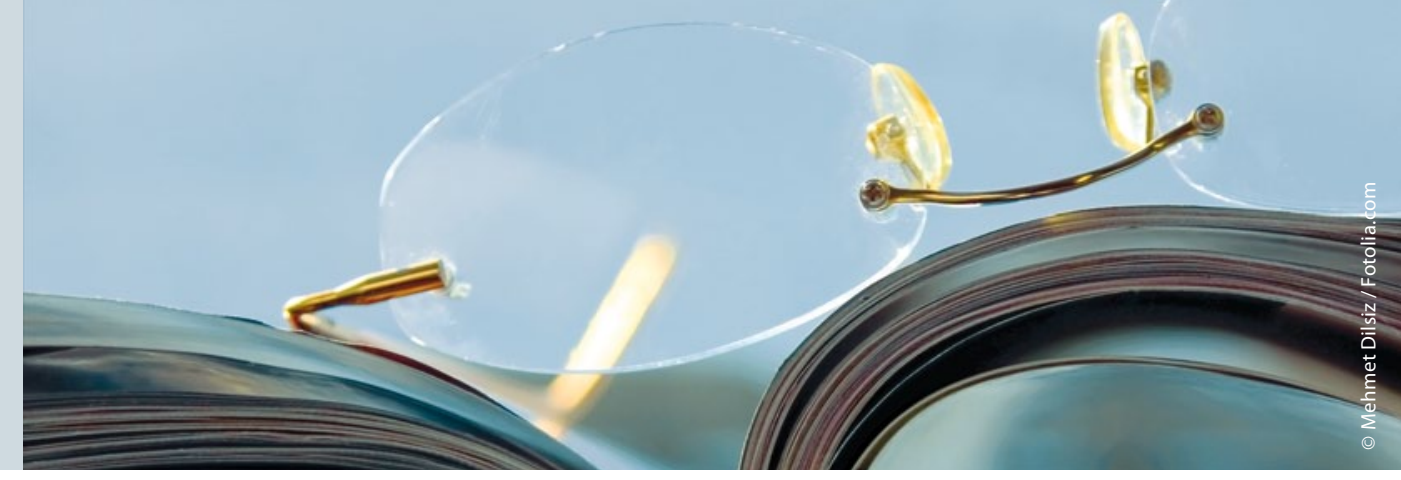

Kommentar: Die Studie bestätigt bisherige Untersuchungen, dass eine intensivierte Senkung des Blutzuckers nach einem Schlaganfall gefährlich sein kann. So wurde in der so genannten NICESUGAR Trial mit über 6.000 intensivpflichtigen Patienten gezeigt, dass eine intensivierte Insulintherapie mit Hypoglykämien verbunden ist. Dies beleuchtet auch den pathophysiolo- gischen Zusammenhang: Durch eine erhöhte Zahl von extremen Hypoglykämien kommt es auch zu einer erhöhten Mortalität. Insofern unterstreicht diese Studie, dass es keine Evidenz für eine intensivierte Insulintherapie bei intensivpflichtigen $\mathrm{Pa}$ tienten gibt. Dies wurde in dieser Studie nun auch für neurologische Patienten mit einem Schlaganfall gezeigt. Es muss aller- dings berücksichtigt werden, dass diese Studie nur eine kleine Fallzahl hatte $(n=180)$ und nur die ersten 24 Stunden nach einem Schlaganfall berücksichtigte.

Professor Stefan Evers

Rosso C et al. Intensive versus subcutaneous insulin in patients with hyperacute stroke: results from the randomized INSULINFARCT trial. Stroke 2012; 43: 2343-2349

\section{Systemische Lysetherapie allein oder mit anschließender lokaler Lysetherapie?}

\section{Studien zeigen, dass die Kombination aus systemischer und lokaler Lyse- therapie weder in punkto Effektivität noch Sicherheit einen Vorteil bringen.}

$Z^{2}$ unehmend wird eine lokale Lysetherapie an eine systemische Lysetherapie mit rt-PA bei mittleren bis schweren Schlaganfällen angeschlossen, es ist aber bis heute noch nicht geklärt, ob diese Kombination wirklich effektiver ist als nur die systemische Lysetherapie alleine. In dieser Studie wurden daher Patienten, die innerhalb von drei Stunden eine systemische Lysetherapie erhielten und die Voraussetzungen für eine lokale Lysetherapie erfüllten, randomisiert entweder mit dieser lokalen Lysetherapie behandelt oder nicht (in einem Randomisierungsverhältnis von $2 \mathrm{zu}$ 1). Der primäre Endpunkt war ein Wert von 2 oder weniger auf der modifizierten Rankin-Skala nach 90 Tagen. Die Studie wurde vorzeitig abgebrochen, nachdem bereits 656 Patienten (434 Patienten mit lokaler Lysetherapie und 222 mit alleiniger systemischer Lysetherapie) randomisiert waren. Der Anteil der Patienten mit einem Wert von 2 oder weniger auf der modifizierten Rankin-Skala nach 90 Tagen unterschied sich nicht signifikant zwischen den beiden Untersuchungsgruppen $(40,8 \%$ mit lokaler Lysetherapie und 38,7\% mit systemischer Lysetherapie). Auch in verschiedenen Subgruppenanalysen (z. B. nach Gruppen eingeteilt gemäß dem Wert auf der NIHStroke Scale) waren keine signifikanten Unterschiede zu beobachten. Die Mortalität war ebenfalls zwischen den beiden Behandlungsgruppen nicht signifikant unterschiedlich (19,1\% vs. $21,6 \%)$ ebenso wie der Anteil der Patienten mit einer symptomatischen intrazerebralen Blutung innerhalb von 30 Stunden nach systemischer Lysetherapie (6,2\% vs. 5,9\%). Zusammenfassend zeigte die Studie also ähnliche Ergebnisse sowohl in der Effektivität als auch in der Sicherheit für die systemische Lysetherapie und für die Kombination mit lokaler Lysetherapie.

Kommentar: Die Ergebnisse dieser Studie sind auf den ersten Blick enttäuschend, legen sie doch nahe, dass eine lokale Lysetherapie nach einer systemischen keinen zu- sätzlichen Benefit für die Patienten bedeutet. Angesichts der Kosten und der Risiken der lokalen Lysetherapie könnte also gefolgert werden, dass dieses Verfahren obsolet ist. Allerdings muss berücksichtigt werden, dass hier alle Fälle, die prinzipiell für eine lokale Lysetherapie in Betracht gekommen sind, zusammengefasst wurden. In vielen Fällen hätte man möglicherweise außerhalb der Studie gar keine lokale Lysetherapie durchgeführt, sodass die statistische Schärfe der Studie nur gering ist. Eine Subgruppe, die von eine zusätzlichen lokalen Lysetherapie profitiert, konnte in dieser Studie nicht identifiziert werden, mag aber existieren, sodass auch in Zukunft in Einzelfällen eine Kombination aus systemischer und lokaler Lysetherapie gerechtfertigt sein mag. Eine unkritische Kombination der Lysetherapien nach dem Motto „viel hilft viel" sollte aber vermieden werden.

Professor Stefan Evers

Broderick JP et al. (The Interventional Management of Stroke (IMS) III Investigators) Endovascular therapy after intravenous t-PA versus t-PA alone for stroke. N Engl J Med 2013 [Epub ahead of print] 